\title{
Organoid variability examined
}

Single-cell transcriptomics is used to determine what cell types are present in brain organoids and how much these cell types vary across organoids.

Given the right conditions, human pluripotent stem cells (hPSCs) can self-organize into $3 \mathrm{D}$ structures that qualitatively resemble human brain tissue. These findings, and similar ones for other tissues, have led to the notion that organoids could be the way forward to study human development, tissue function, and disease-all in a dish.

But how good are organoids as models of in vivo tissue? To assess this, one needs to know how closely their cell types match those found in vivo. One must also determine how this cell-type distribution varies from organoid to organoid, from hPSC line to hPSC line, and from one differentiation batch to another. Giorgia Quadrati and Paola Arlotta at Harvard University set out to tackle some of these questions.

Starting with a human induced PSC line and culture conditions optimized and refined from previously reported ones, the researchers grew brain organoids via an unpatterned protocol for up to 9 months. "We wanted to use a system where the investigator interferes as little as possible," says Arlotta of the decision to use methods that do not explicitly pattern the cells. At 3 and 6 months, they then measured the singlecell transcriptome of a total of about 80,000 cells from 31 organoids using the dropletbased Drop-seq approach.

What they saw, says Arlotta, was a surprise. "We were blown away," she says, "by the diversity of cells and the fidelity of cell identity." After 6 months of culture, the transcriptomic signatures of almost 70,000 cells in brain organoids could be grouped into ten clusters, seven of which had a recognizable identity when compared to existing data sets. Six of these clusters resembled neuroectodermal cells; they included cells resembling forebrain neurons, retinal cells, astrocytes and neural progenitors. When the forebrain and retinal expression signatures were dissected further and compared to single-cell data either from adult mouse retina or from human fetal cortex, the researchers saw a good correlation between the cellular subtypes in organoids and in vivo tissue.

While most of the identified cell types were present in almost all of nineteen 6-month-old organoids, it should be noted that some (forebrain neurons for example) were only seen in about one-third of them. It will be necessary to take such variability into account when using this system to study, for example, the effect of a perturbation on a given cell type.

Batch effects, Arlotta and colleagues report, are part of the story. "We have seen again and again that when you have a successful bioreactor, [the bioreactor] is successful," says Arlotta. In other words, the organoids from some bioreactors (or differentiation batches) all generate forebrain or retinal tissue, but there are other bioreactors where that is not the case. Better understanding and control of what makes a 'successful' bioreactor, or an ability to identify these early in the process, will be important for the efficient use of organoids as models. There is also variability between hPSC lines, Arlotta points out, as has been seen for 2D differentiation.

It should be noted that, by looking at an average of 3,500 cells per organoid, the researchers are substantially undersampling the cells per organoid. This could mean that the variability they report is an overestimate. There is also the possibility that additional cell types in these structures have been missed.

Arlotta sees organoids as the only system available today to study many human cell types. What they are not good enough for yet is to study circuits, she says, because their anatomy and the relative positioning and connections of cells may not be quite right compared to in vivo tissue. But she sees this as an opportunity. "Nature can build it, let's begin to control it," she says. "My hope is that more and more developmental neurobiologists will enter this field and make it happen."

Natalie de Souza

RESEARCH PAPERS

Quadrato, G. et al. Cell diversity and network dynamics in photosensitive human brain organoids. Nature 545, 48-53 (2017). 